\title{
:
}

\section{Intentional Binding and Sense of Agency of Visual Outcomes}

Fatemeh Ansarinejad $^{1}$, Alireza Moradi ${ }^{*}$, Reza Khosrowabadi ${ }^{3}$, Ali Fathi Ashtiani ${ }^{4}$

${ }^{1}$ Department of Clinical Psychology, University of Science and Culture, Tehran, Iran

${ }^{2}$ Department of Clinical Psychology, Faculty of Psychology and Educational Sciences, Kharazmi University, Tehran, Iran

${ }^{3}$ Department of Cognitive Sciences and Brain, Shahid Beheshti University, Tehran, Iran

${ }^{4}$ Department of Behavioral Sciences, Baqiyatallah University of Medical Sciences, Tehran, Iran

\section{ABSTRACT}

Introduction: The interval between voluntary actions and their outcomes are perceived as shorter than the interval between similar involuntary actions and the outcomes. This bias in time perception may be related to the sense of agency which has been referred to as intentional binding (IB). In this study, we investigated the effect of IB on voluntary action and its visual outcome (ie. color changing to red, green, blue, and yellow). Besides, we compared the IB effects among these different colors. Materials and Methods: 19 right-handed subjects (14 women and 5 men) have participated in baseline and experimental studies. Participants should press a key and after that, they would face color-change, then they reported the time perceptions, via referring to Libet's clock. These data were compared to the baseline data (pressing key without color-change and color-change without pressing the key). We modified Libet's clock in a new version to study the IB of visual effect. Results: The results show that the total IB (sum of action IB and color IB) in overall and distinct colors was significantly different. Action IB was not significantly different, however, the happening of the color IB in overall and distinct colors was significantly different. Furthermore, a significant difference between action IB - blue outcomes was observed compared to action IB - green and yellow outcomes. There were no significant differences among different color IB. Conclusion: Using innovative Libet's clock, we could investigate and compare IB of visual outcome in 4 different colors. Our results have been in line with previous studies and with the retrospective viewpoint of IB phenomena.

\section{Keywords:}

1. Intention

2. Time Perception

3. Color

\section{*Corresponding Author: Alireza Moradi}

E-mail:moradi@khu.ac.ir 
(قصد -بستخى) و (حس عامليت) يبى آمدهاى ديدارى

\author{
فاطمه انصارىنزاد'، عليرضا مرادى זّ،: رضا خسرو آبادى"، على فتحى آشتيانى ‘

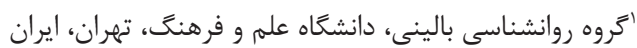

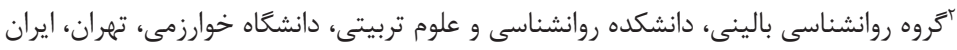

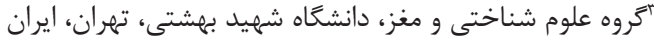

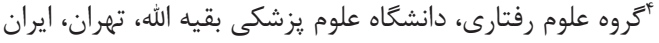

دريافت: F ش شهريور qجس|

\begin{tabular}{|c|c|}
\hline 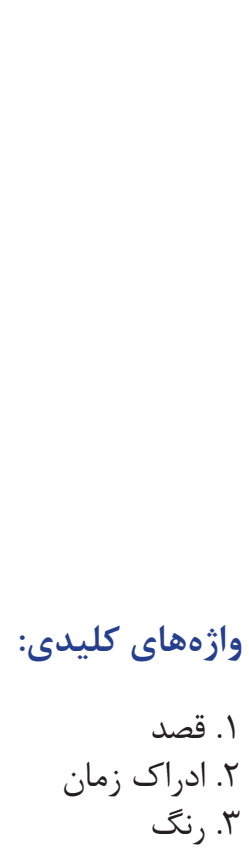 & 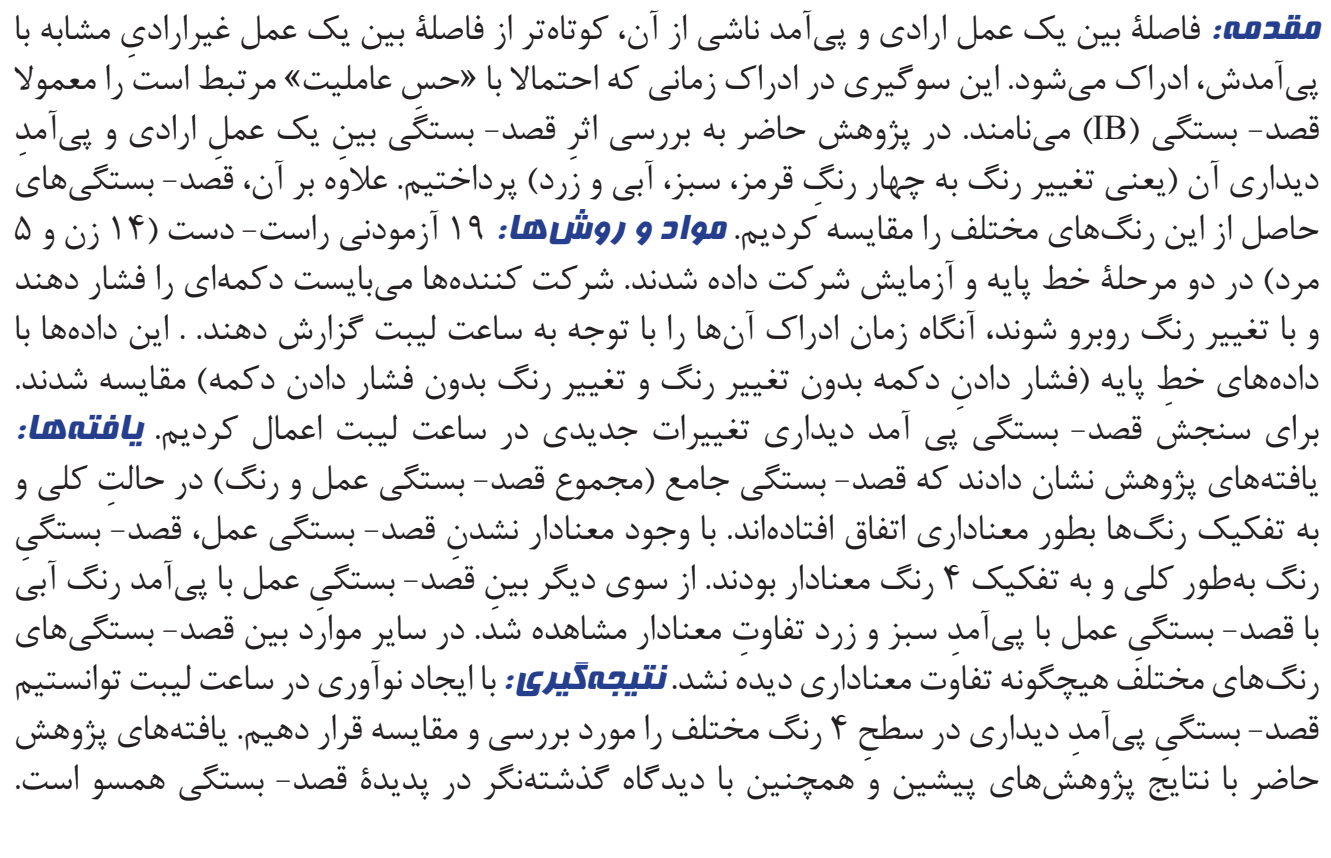 \\
\hline
\end{tabular}

*نويسنده مسئول: عليرضا مرادى يست الكترونيك: moradi@khu.ac.ir 


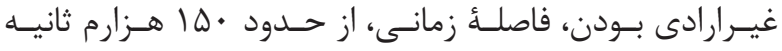

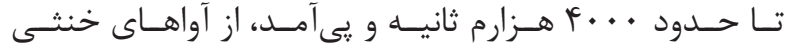

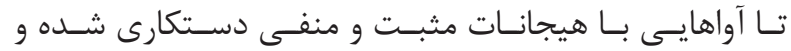

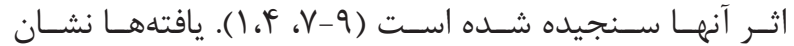

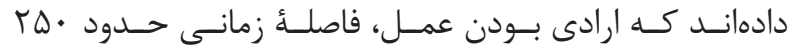

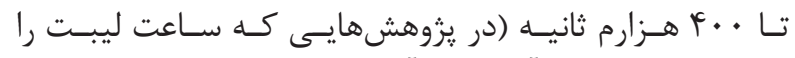

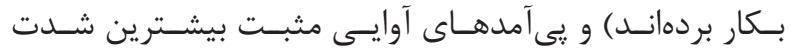

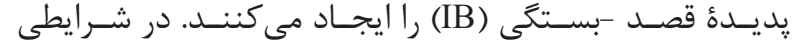

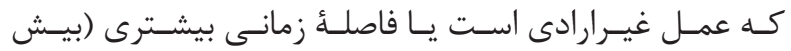

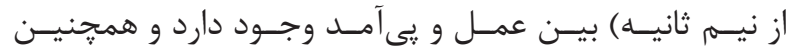

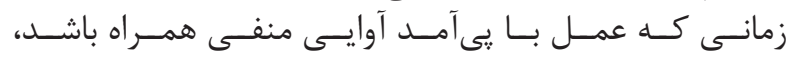

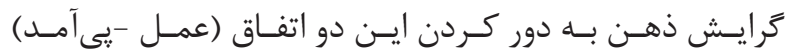

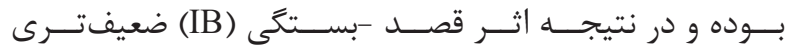

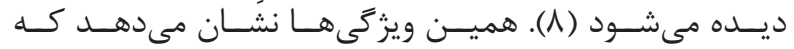

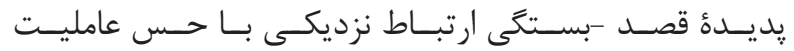

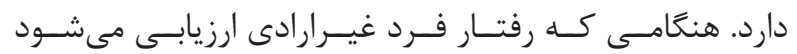

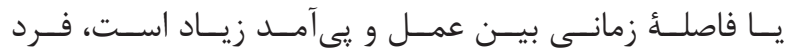

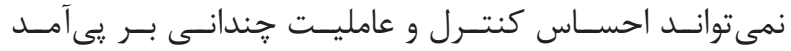

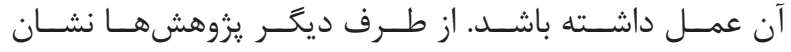

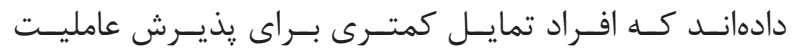

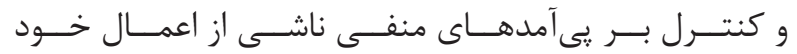

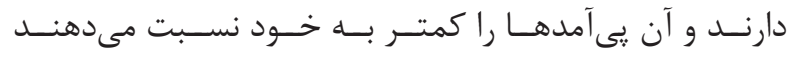

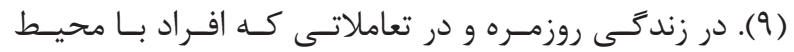

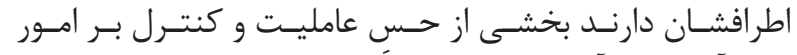

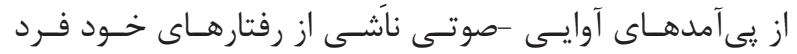

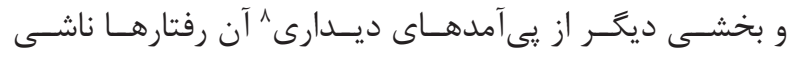

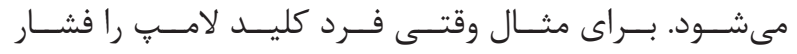

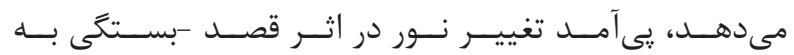

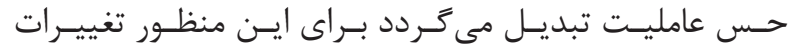

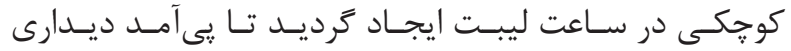

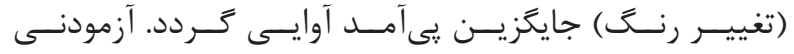

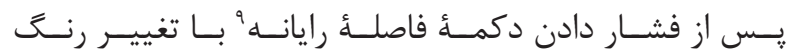

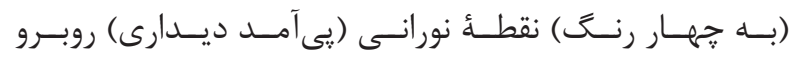

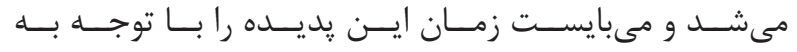

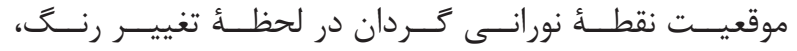

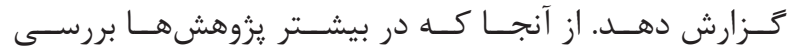

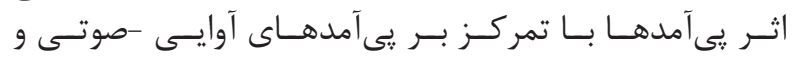

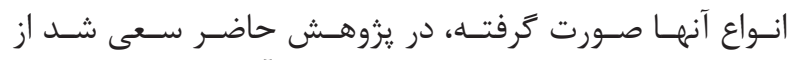

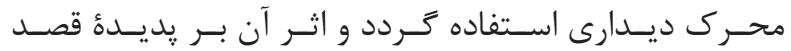

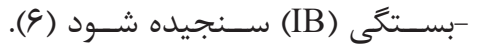

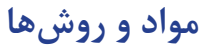

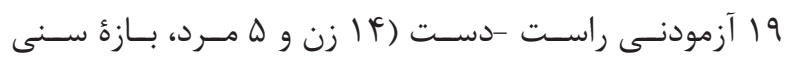

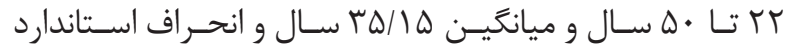

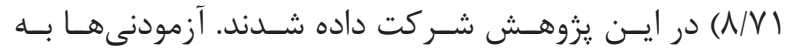

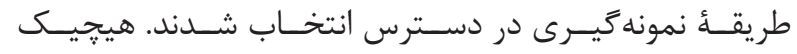

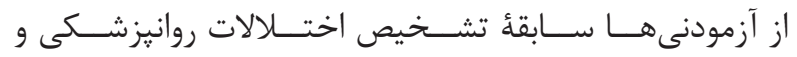

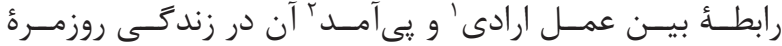

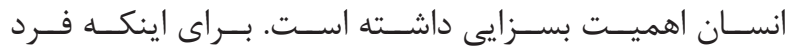

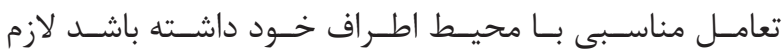

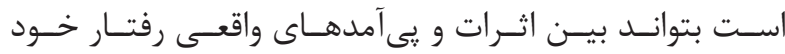

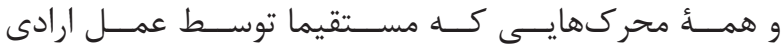

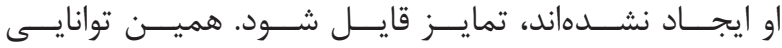

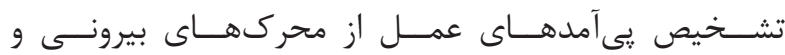

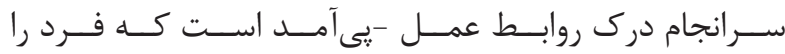

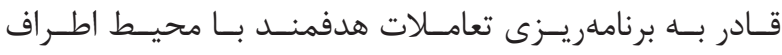

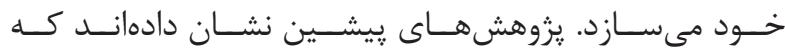

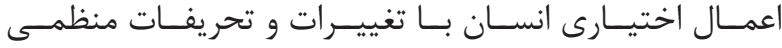

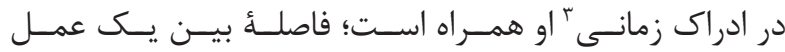

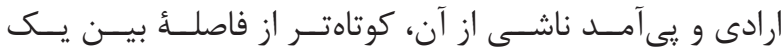

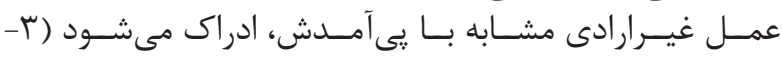

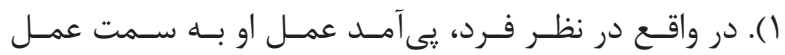

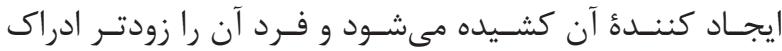

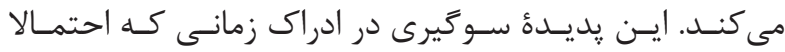

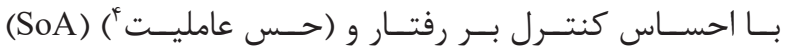

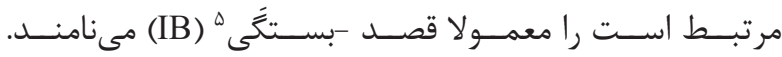

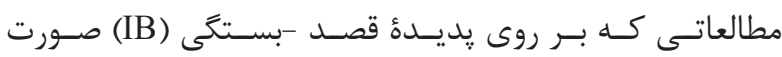

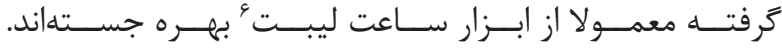

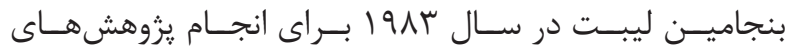

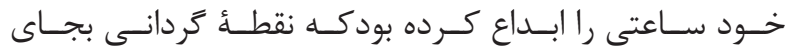

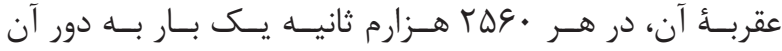

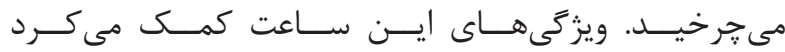

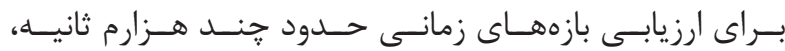

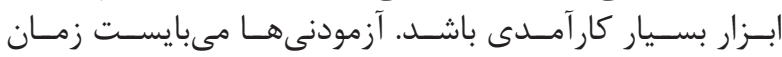

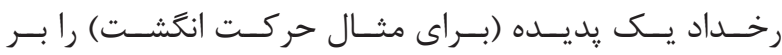

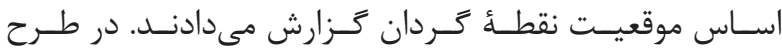

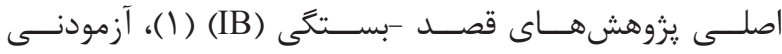

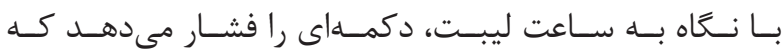

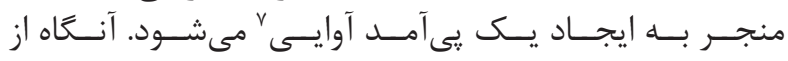

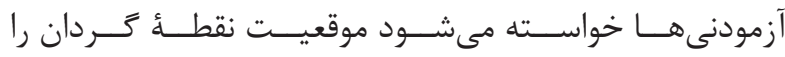

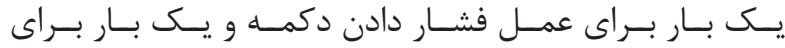

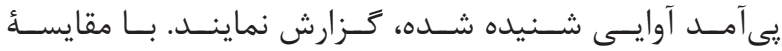

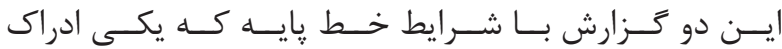

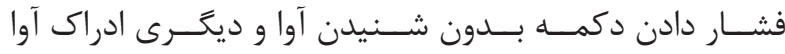

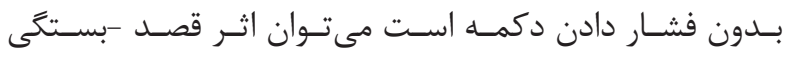

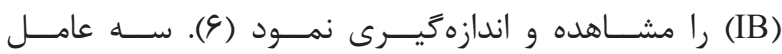

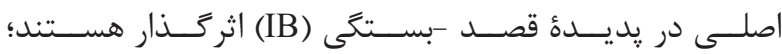

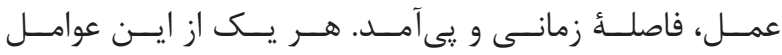

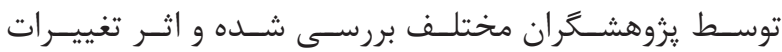

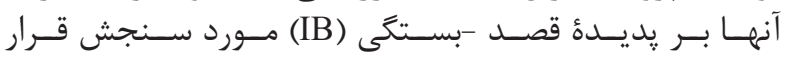

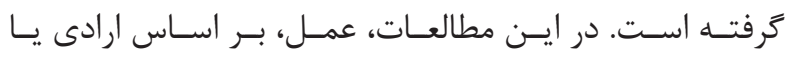




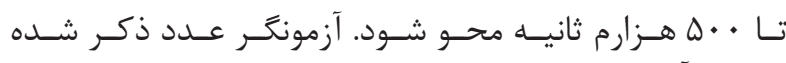

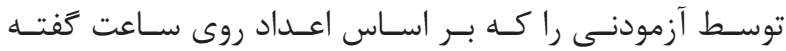

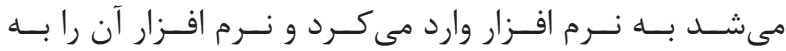

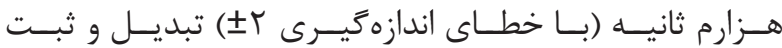

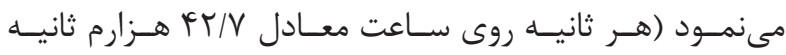

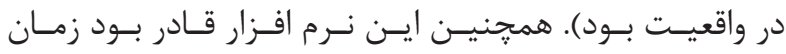

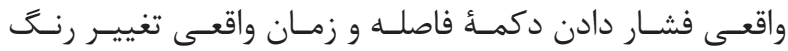

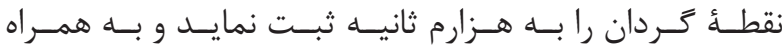

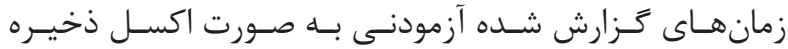

نمايـد.

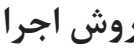

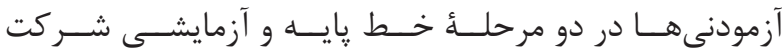

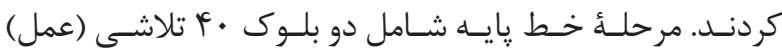

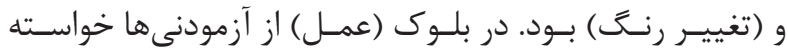

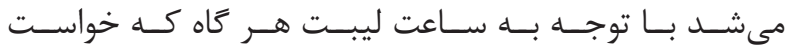

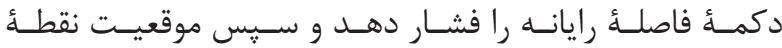

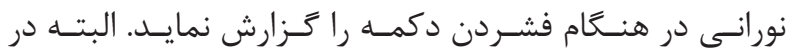

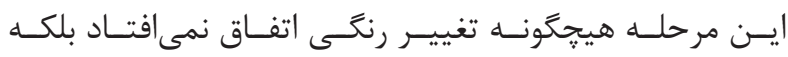

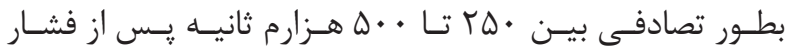

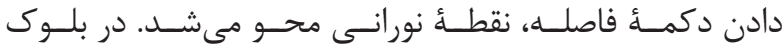

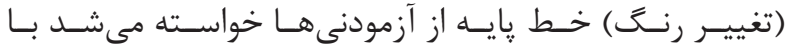

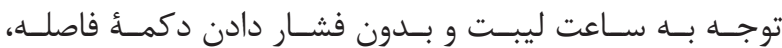

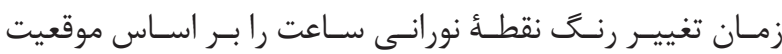

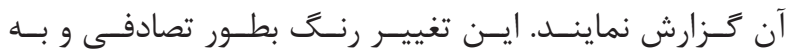

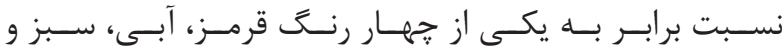

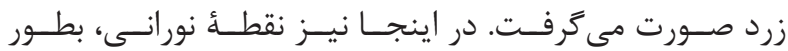

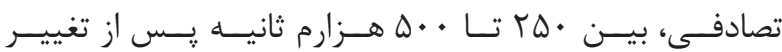

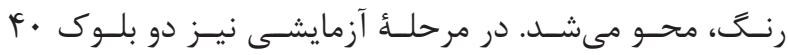

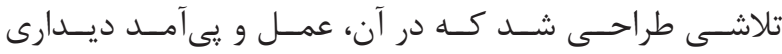

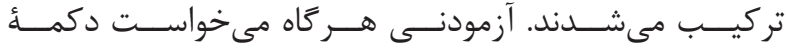

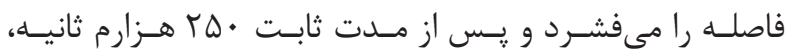

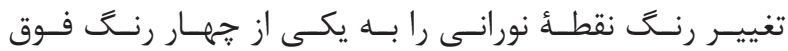

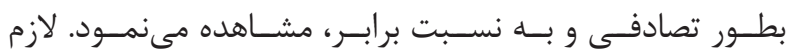

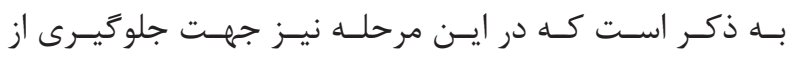

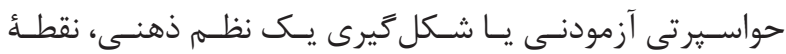

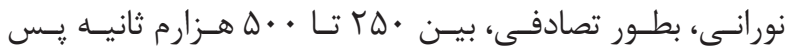

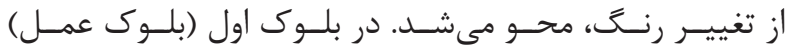

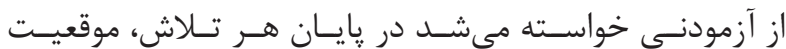

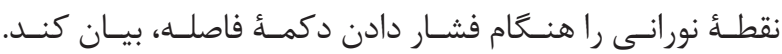

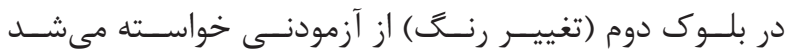

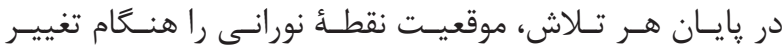

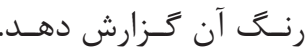

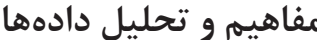

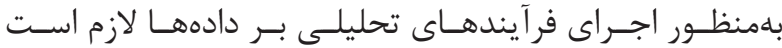

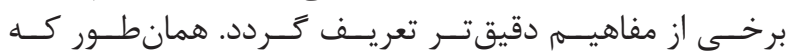

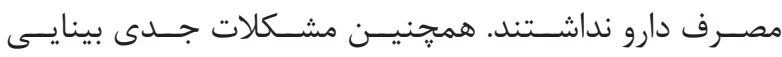

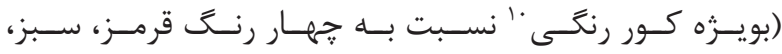

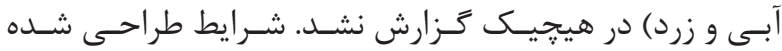

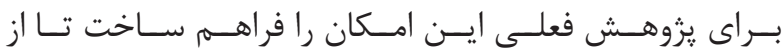

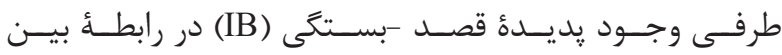

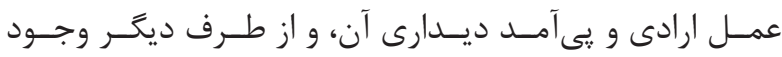

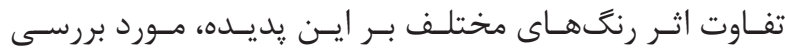

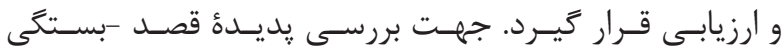

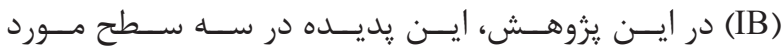

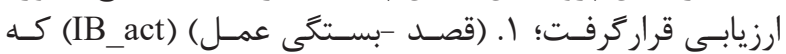

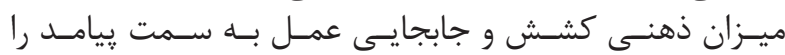

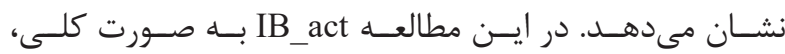

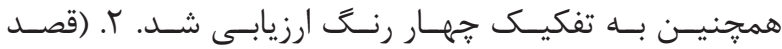

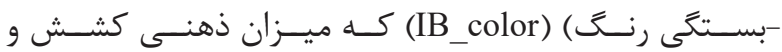

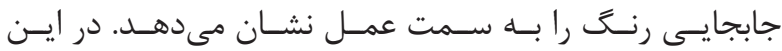

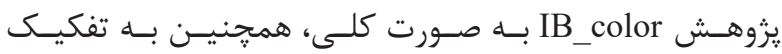

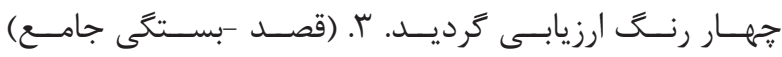

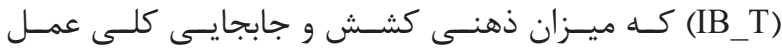

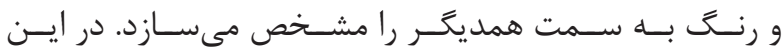

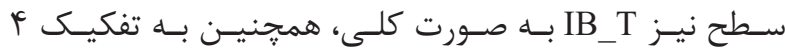

$$
\text { رنـَ ارزيابـى شـد. }
$$

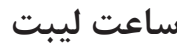

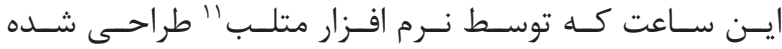

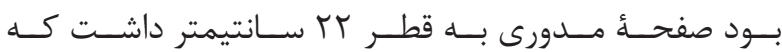

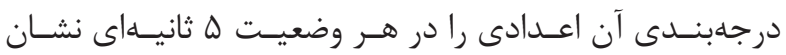

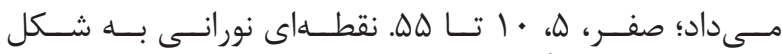

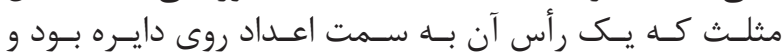

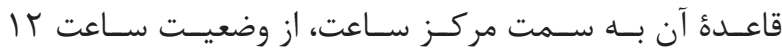

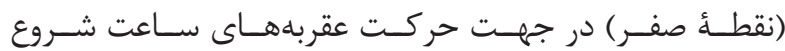

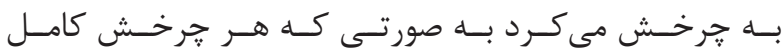

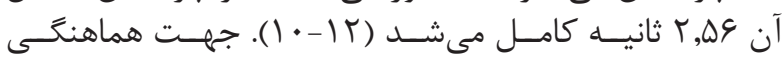

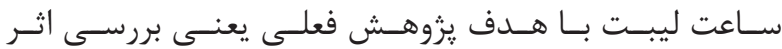

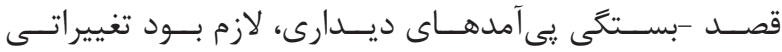

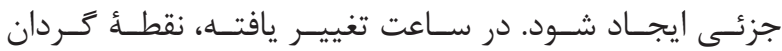

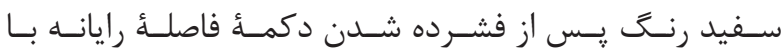

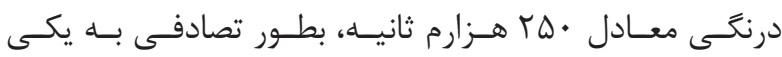

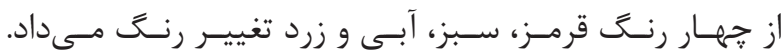

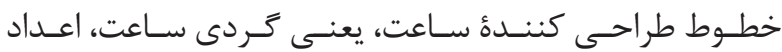

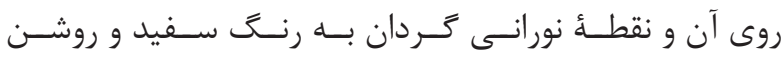

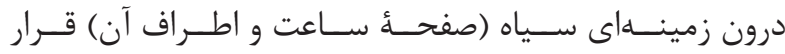

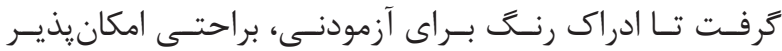

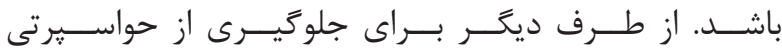

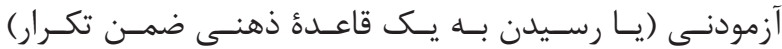

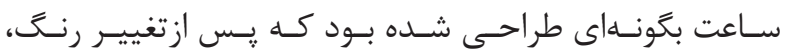

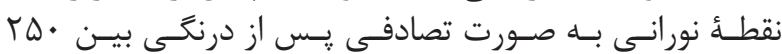




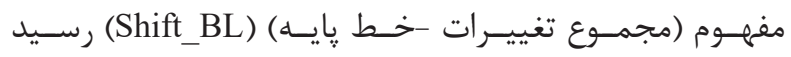

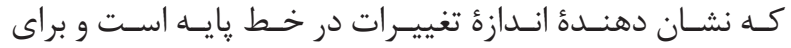

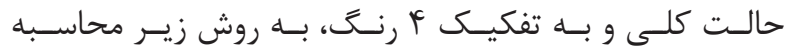

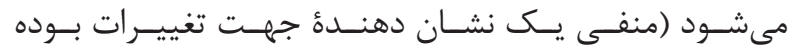

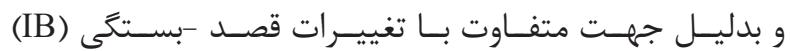
لحــاظ شـده اســت $1-\left(^{*}\right)$ Color $_{(4)-B L(-) A c t \_B L}=\left(\right.$ Shift $\left._{(4)-B L}\right)$

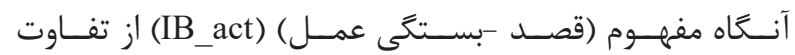

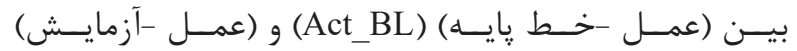
بدسـت آمــــ (Act_exp)

Act_exp(-)Act_BL $=\left(\mathrm{IB}_{(4)-}\right.$ act $)$

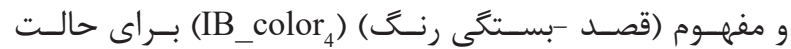

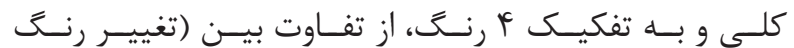

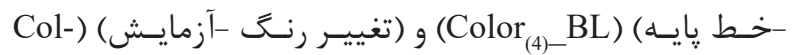
Color $_{(4)-} \exp (-)$ Color $_{(4)-B L}=\left(\mathrm{IB} \_\right.$color $\left.{ }_{4}\right)$ (or (ou $_{(4)}$ exp

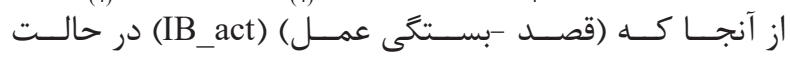

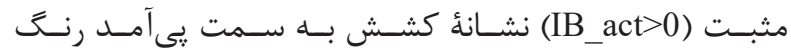

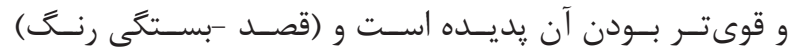
(IB_color $\left.{ }_{4}\right)$

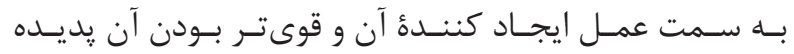

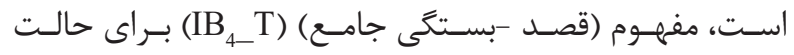

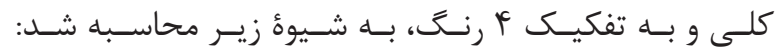
IB_color ${ }_{4}(-) \mathrm{IB} \_$act $=\left(\mathrm{IB}_{(4)-} \mathrm{T}\right)$

بافتهها

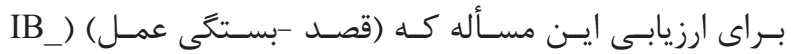
(act

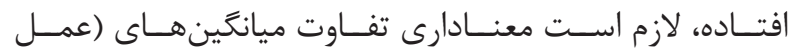

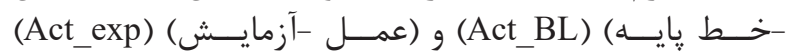

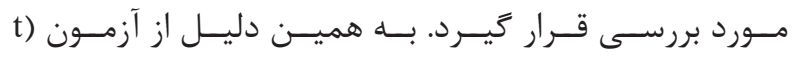

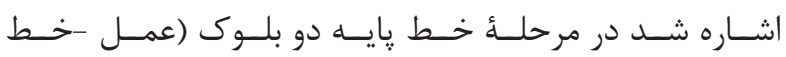

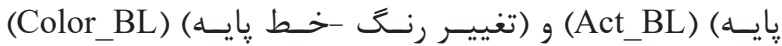

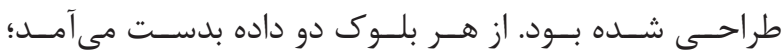

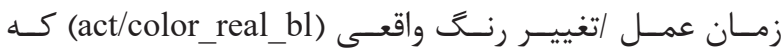

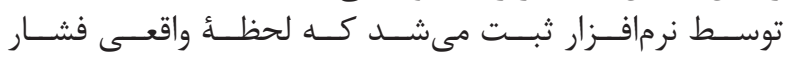

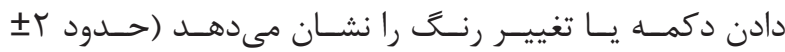

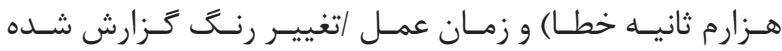

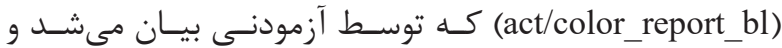

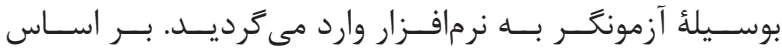

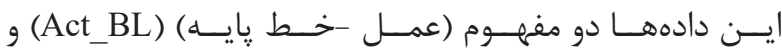

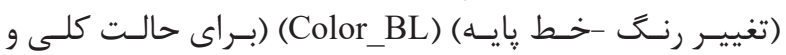

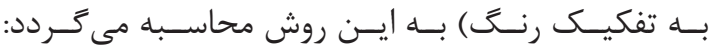
act_report_bl(-)act_real_bl=(Act_BL) color $_{(4)-}$ report_bl(-)color ${ }_{(4)-}$ real_bl $=\left(\right.$ Color $\left._{(4)-B L}\right)$

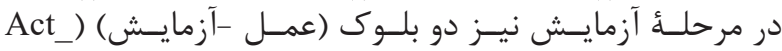
(exp

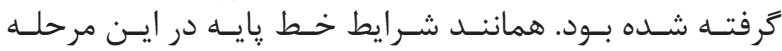

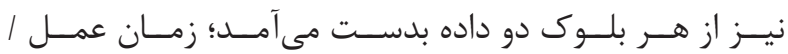

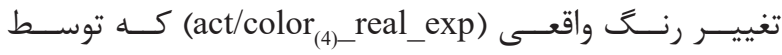

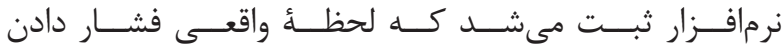

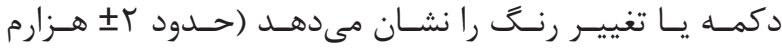

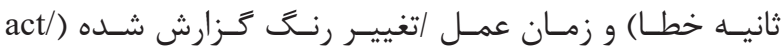
(color report_exp

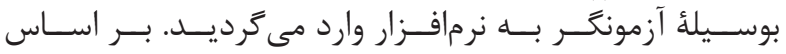

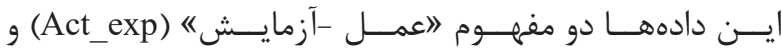

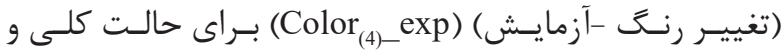

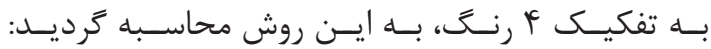
act_report_exp(-)act_real_exp=(Act_exp)

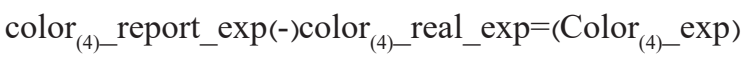

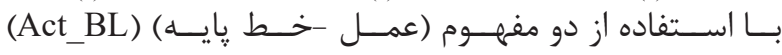

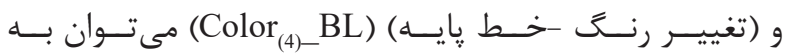

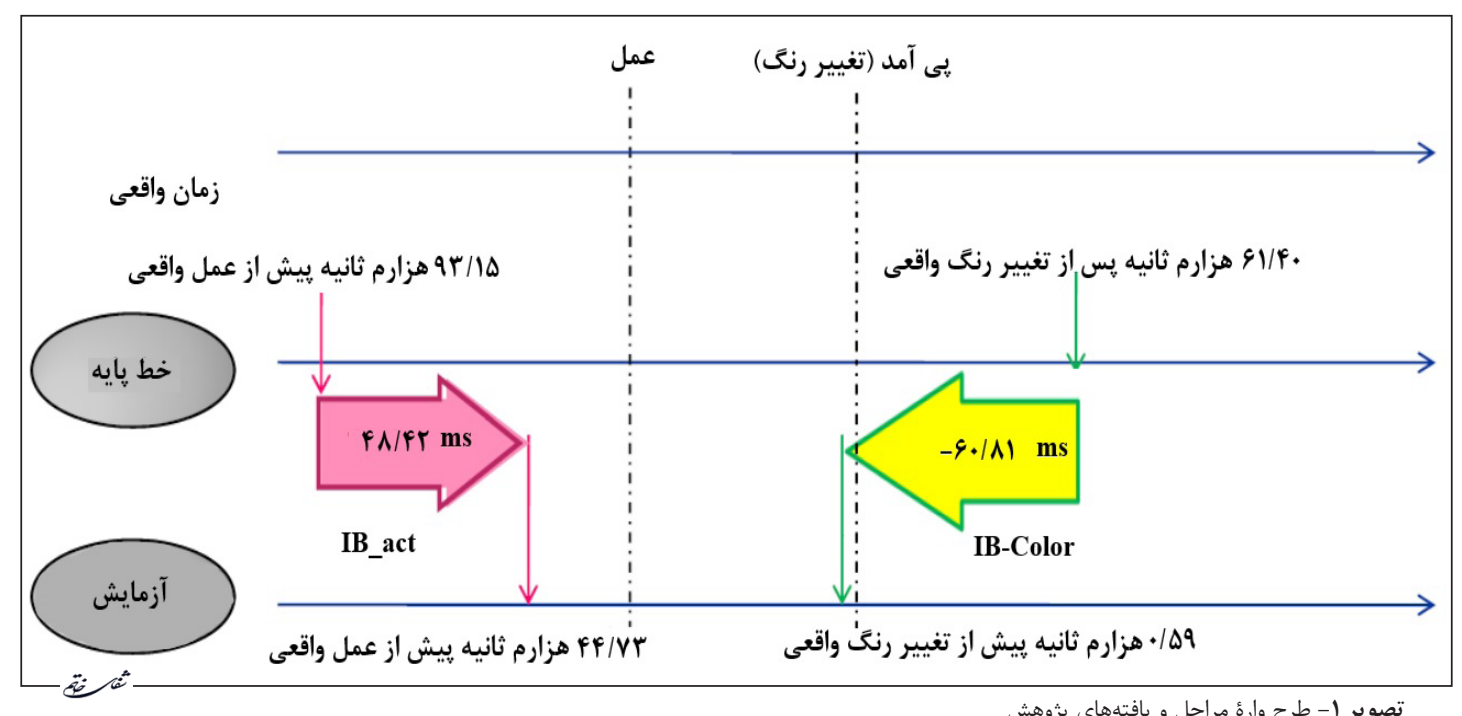

تصوير ا- طرح وارهٔ مراحل و يافتههاى يثوهش 


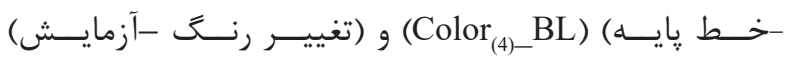

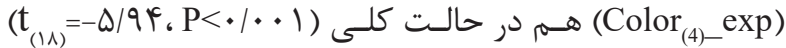

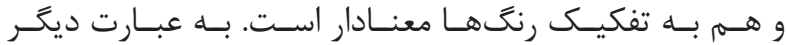

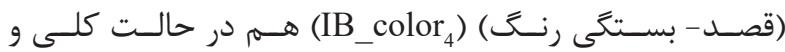

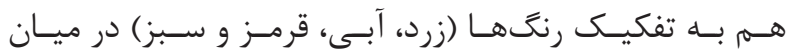

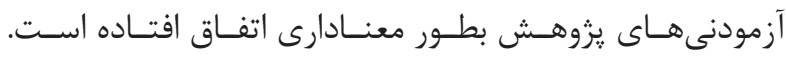
بــه جهـت ارزيابـى ايـن مســأله كـه (قصـد -بسـتخى جامـع )

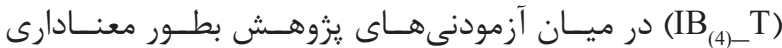

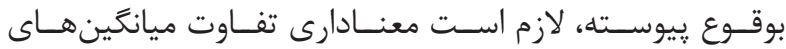

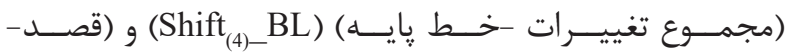

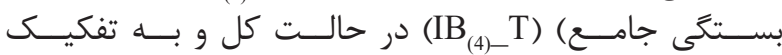

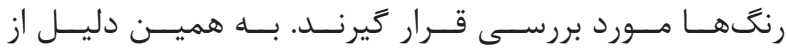

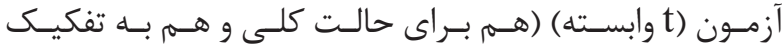

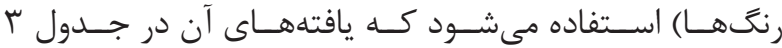

وابسـته) اسـتفاده شـد كـهـ يافتههـاى آن در جــدول ا آمـده

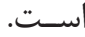

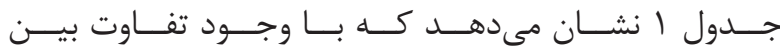

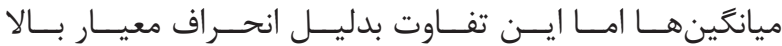

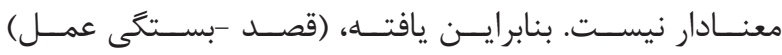

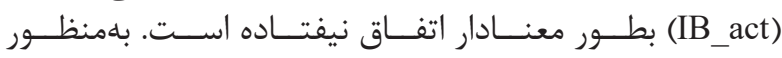

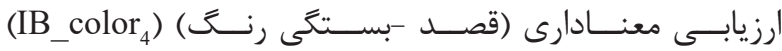

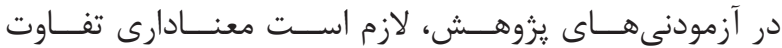

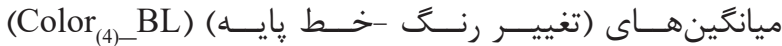

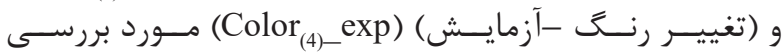

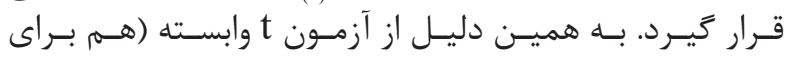

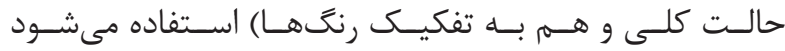

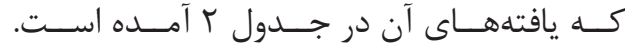

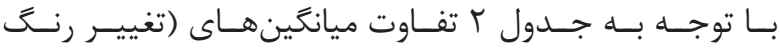

جدول ا- آزمون تفاوت ميانكَينها بين مرحلة خط هايه و آزمايش

\begin{tabular}{|c|c|c|c|c|c|c|}
\hline $\mathbf{P}$ & $\mathbf{T}$ & DF & انحراف معيار & ميانگين & مرحله & متغير \\
\hline \multirow{2}{*}{.1 .94} & \multirow{2}{*}{ I/VA } & \multirow{2}{*}{11} & $11 \mathrm{r} / \Delta 9$ & $94 / 109$ & خط پايـ & \multirow{2}{*}{ عمل } \\
\hline & & & $V \boldsymbol{F} / \cdot \boldsymbol{r}$ & FF/VTN & آزمايش & \\
\hline
\end{tabular}

جدول r - يافتهاى آزمون تفاوت ميانگًنها بين خط پِايه و آزمايش

\begin{tabular}{|c|c|c|c|c|c|c|}
\hline $\mathbf{P}$ & $\mathrm{T}$ & DF & أحراف معيار & ميانكين & مر حله & متغير \\
\hline \multirow{2}{*}{$.1 \cdot \cdot 1$} & \multirow{2}{*}{$-\Delta / q \mathcal{F}$} & \multirow{2}{*}{11} & $r q / q$. & $-q \cdot / 4$. & خط يايه & \multirow{2}{*}{ تغيير رنگ كل } \\
\hline & & & $\Delta Y / V T$ & -.109 & آزمايش & \\
\hline \multirow{2}{*}{.1 .94} & \multirow{2}{*}{$-r / l$} & \multirow{2}{*}{11} & Fr/pte & -GY/Dr & لـ پايه & \multirow{2}{*}{ زرد } \\
\hline & & & $V V / \Lambda F$ & $-11 / 9 V$ & & \\
\hline \multirow{2}{*}{$\cdot 1 \cdot \cdot 1$} & \multirow{2}{*}{$-\omega / \cdot r$} & \multirow{2}{*}{11} & $\Delta G / \Lambda G$ & $-\Delta \cdot / V c$ & خط پپايه & \multirow{2}{*}{ آبى } \\
\hline & & & cY/VV & $11 / \Delta F$ & آزمايش & \\
\hline \multirow{2}{*}{$.1 \cdot \cdot 1$} & \multirow{2}{*}{$-f / f}$. & \multirow{2}{*}{11} & $F \Delta / V q$ & $-9 \cdot 19 r$ & خط ڤֶايه & \multirow{2}{*}{ قرمز } \\
\hline & & & $\Delta V / V D$ & $V / .9$ & آزمايش & \\
\hline \multirow{2}{*}{$\cdot 1 \cdot \cdot 1$} & \multirow{2}{*}{$-\Delta / 1 f$} & \multirow{2}{*}{11} & FI/Tr & $-৭ q / V$ & خط پايه & \multirow[b]{2}{*}{ سبز } \\
\hline & & & 0.194 & $-9 / 4$. & آزمايش & \\
\hline
\end{tabular}


معنــادارى تفاوتهــاى آنهــا در جــدول f آمــده اسـت:

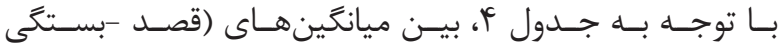

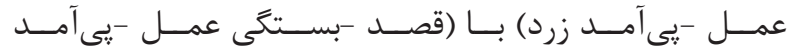

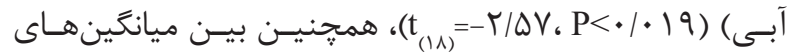

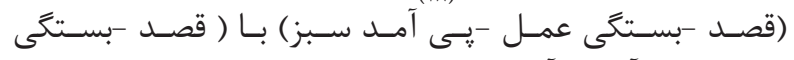

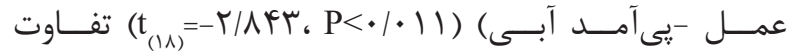

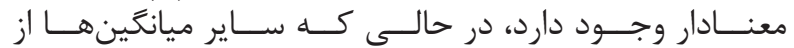

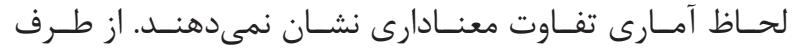

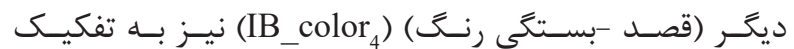

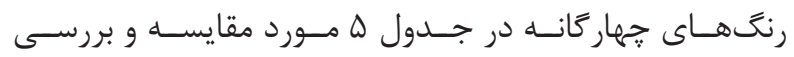

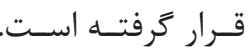

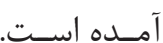

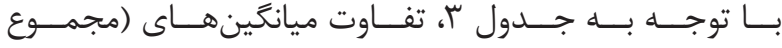

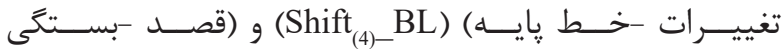

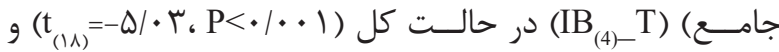

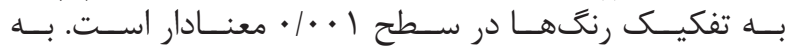

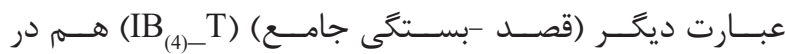

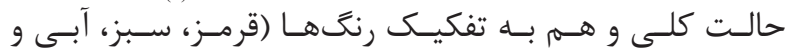

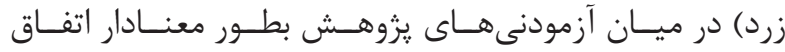

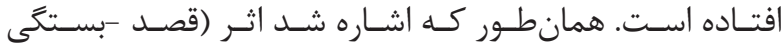

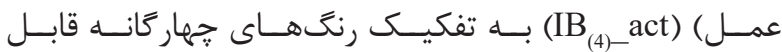

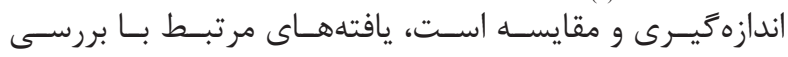

جدول بـ يافتههاى آزمون تفاوت ميانكَينها بين خط پايه و آزمايش

\begin{tabular}{|c|c|c|c|c|c|c|}
\hline $\mathbf{P}$ & $\mathbf{T}$ & DF & انحراف معيار & ميانكًين & مرحله & متغير \\
\hline \multirow[t]{2}{*}{$\cdot 1 \cdot \cdot 1$} & $-Q / \cdot r$ & 11 & $\mid r F / \cdot q$ & $-|f \Delta /| f$ & خط پايه & \multirow{2}{*}{ مجموع تغييرات كل قصد -بستگى جامع كل } \\
\hline & & & $119 / 4 T$ & 1.9/Tr & آزمايش & \\
\hline \multirow[t]{2}{*}{$\cdot 1 \cdot \cdot 1$} & $-f / q p$ & 11 & $11 \cdot / A r$ & $-\mid \Delta H / V T$ & خط پِايه & \multirow{2}{*}{ مجموع تغييرات زرد قصد -بستگى جامع زرد } \\
\hline & & & $|D| / V F$ & $91 / 99$ & آازمايش & \\
\hline \multirow[t]{2}{*}{$\cdot 1 \cdot \cdot 1$} & $-\Delta / \mathcal{F V}$ & 11 & $1 \cdot 9 / 91$ & - IET/Ke & خط پايه & \multirow{2}{*}{ مجموع تغييرات آبى قصد -بستكى جامع آبى } \\
\hline & & & $|r| / F \Delta$ & $1 \cdot 4 / \cdot 9$ & آزمايش & \\
\hline \multirow[t]{2}{*}{$\cdot 1 \cdot \cdot 1$} & $-\Delta / \cdot \wedge$ & 11 & Ir./Irt & $-\mid F T / q T$ & خط پايه & \multirow{2}{*}{ مجموع تغييرات قرمز قصد -بستگى جامع قرمز } \\
\hline & & & ITH/VD & $1 \% \cdot / \Lambda V$ & 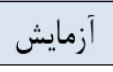 & \\
\hline$\cdot 1 \cdot \cdot 1$ & $-\omega / \mu \cdot$ & 11 & $1 \cdot F / V 1$ & $-1 \Delta V / \varepsilon \Psi$ & خط پِايه & \multirow{2}{*}{ مجموع تغييرات سبز قصد -بستكى جامع سبز } \\
\hline ثاث & & & $|r \Delta / \Gamma|$ & $1 \cdot \mu / \cdot$ & آزمايش & \\
\hline
\end{tabular}

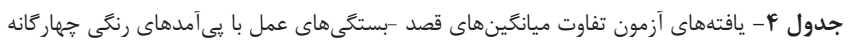

\begin{tabular}{|c|c|c|c|c|c|}
\hline $\mathbf{P}$ & $\mathbf{T}$ & DF & انحراف معيار & ميانگين & متغير ها \\
\hline \multirow[t]{2}{*}{.1 .19} & $-r / \Delta V$ & 11 & $111 / 99$ & $\Delta \cdot / 19$ & قصل -بستگى عمل (زرد) \\
\hline & & & $11 Q / \cdot F$ & GN/DG & قصد -بستگى عمل (آبى) \\
\hline \multirow[t]{2}{*}{.1 .90} & I/VG & 11 & $110 / \cdot F$ & GN/DS & قصد -بستگى عمل (آبى) \\
\hline & & & $\mid f V / 199$ & r & قصد -بستىى عمل (قرمز) \\
\hline \multirow[t]{2}{*}{ • } & $-\cdot 19$ & 11 & $\mid f V / 19$ & r l/rq & قصد -بستى عمل (قرمز) \\
\hline & & & IrT/IV & FT/GD & قصد -بستخى عمل (سبز) \\
\hline \multirow[t]{2}{*}{$\cdot \ln$} & $-\cdot / V q$ & 11 & IrT/IV & FT/ & قصد -بستگى عمل (سبز) \\
\hline & & & $111 / 99$ & $0 \cdot 119$ & قصد -بستخى عمل (زرد) \\
\hline \multirow[t]{2}{*}{$\cdot / \mu F$} & .191 & 11 & $111 / 99$ & $0 \cdot 119$ & قصل -بستگى عمل (زرد) \\
\hline & & & $1 F V / 19$ & $r 1 / r q$ & قصد -بستىى عمل (قرمز) \\
\hline \multirow[t]{2}{*}{$.1 \cdot 11$} & - r/AFr & 11 & $\mid r T / I V$ & Fr/GD & قصد -بستخى عمل (سبز) \\
\hline & & & $\mid 10 / \cdot F$ & GN/DG & قصد -بستخى عمل (آبى) \\
\hline
\end{tabular}




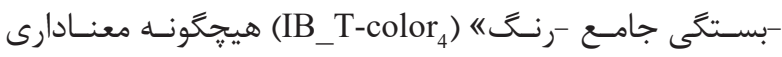

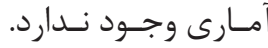
بحث و نتيجه تيرى

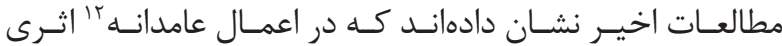

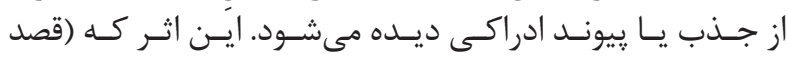

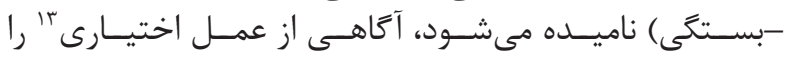

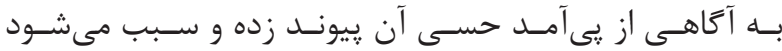

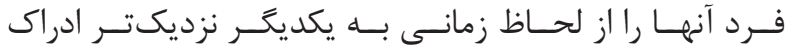

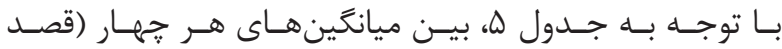
-بسـتخَى رنــَ) (IB_color

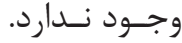

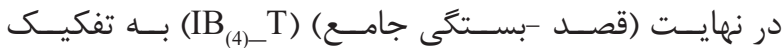

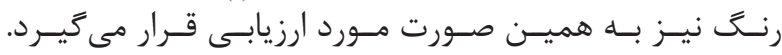

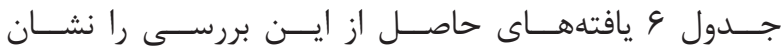
مى دهـــد.

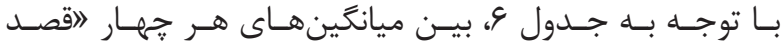

جدول هـ يافتهاى آزمون تفاوت ميانگَينهاى قصد -بستخىهاى رنگ

\begin{tabular}{|c|c|c|c|c|c|}
\hline $\mathbf{P}$ & $\mathbf{T}$ & DF & انحر اف معيار & ميانگين & متغيرهـا \\
\hline \multirow{2}{*}{ - 1949 } & \multirow{2}{*}{ מצואן } & \multirow{2}{*}{11} & $V F / \cdot \Lambda$ & $-\Delta T / \Lambda F$ & قصد -بستخى رنگ زرد \\
\hline & & & $\Delta r / 9$. & - & قصد -بستخى رنگ آبى \\
\hline \multirow{2}{*}{$\cdot / 1 \cdot V$} & \multirow{2}{*}{ - ITFA } & \multirow{2}{*}{11} & $\Delta r / q$. & - & قصد -بستخى رنگ آبى \\
\hline & & & $Q \mathrm{R} / \cdot \mathrm{r}$ & $-9 V / 99$ & قصد -بستخى رنگ قرمز \\
\hline \multirow{2}{*}{$\cdot / 4 q \wedge$} & \multirow{2}{*}{$-\cdot 1991$} & \multirow{2}{*}{11} & $q \mathrm{~d} / \cdot \mathrm{r}$ & $-9 V / 99$ & قصد -بستخَى رنَ قرمز \\
\hline & & & $\Delta 1 / 1 \Delta$ & $-q \cdot / \mathcal{F}$ & قصل -بستخى رنگ سبز \\
\hline \multirow{2}{*}{ r } & \multirow{2}{*}{-. } & \multirow{2}{*}{11} & $\Delta / / 1 \Delta$ & $-\varphi \cdot / 4$ & قصد -بستخًى رنگ سبز \\
\hline & & & $V F / \cdot \Lambda$ & $-\Delta T / A F$ & قصل -بستخى رنغ زرد \\
\hline \multirow{2}{*}{$\cdot / T \Delta R$} & \multirow{2}{*}{$1 / 190$} & \multirow{2}{*}{11} & $V F / \cdot \Lambda$ & $-\Delta T / \Lambda F$ & قصد -بستخى رنگ زرد \\
\hline & & & $q \mathrm{~V} / \cdot \mathrm{r}$ & $-9 V / 99$ & قصد -بستخً رنگ قرمز \\
\hline \multirow{2}{*}{$.191 \mathrm{~F}$} & \multirow{2}{*}{$\cdot 11 \cdot 9$} & \multirow{2}{*}{11} & $\Delta r / 9$. & - & قصد -بستخى رنگ آبى \\
\hline & & & $\Delta / / 10$ & $-q \cdot / \mathcal{E}$ & قصل -بستخى رنخ سبز \\
\hline
\end{tabular}

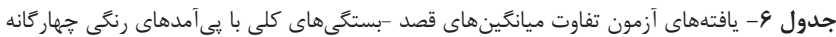

\begin{tabular}{|c|c|c|c|c|c|}
\hline $\mathbf{P}$ & $\mathbf{T}$ & DF & انحراف معيار & ميانگين & متغيرها \\
\hline \multirow[t]{2}{*}{. } & I/TAF & 11 & $\mid r \Delta / 1$ & $|\cdot|+\cdot$ & قصد -بستَّى جامع -زرد \\
\hline & & & Ir/VD & Ir.IAV & قصل -بستخى جامع -آبى \\
\hline \multirow[t]{2}{*}{$\cdot / r \Lambda$} & $-1 / 11 f$ & 11 & Ir/VD & Ir.IAV & قصل -بستخى جامع -آبى \\
\hline & & & $|\Delta| / V Y$ & $91 / 99$ & قصد -بستخى جامع -قرمز \\
\hline \multirow[t]{2}{*}{$\cdot \mid \Lambda F$} & $-\cdot / r \cdot \Delta$ & 11 & $|D| / V F$ & $91 / 99$ & قصد -بستگى جامع -قرمز \\
\hline & & & $|r| / C \Delta$ & $1 \cdot \boldsymbol{c} / \cdot \Delta$ & قصل -بستى جامع -سبز \\
\hline \multirow[t]{2}{*}{.1949} & .1 .99 & 11 & $|r| / F \Delta$ & $|\cdot|+\Delta$ & قصل -بستخى جامع -سبز \\
\hline & & & $|r \Delta / T|$ & $1 \cdot r / \cdot$ & قصل -بستخى جامع -زرد \\
\hline \multirow[t]{2}{*}{$\cdot$ - IAqF } &.$/ I V F$ & 11 & $\mid r \Delta / T_{1}$ & $1 \cdot r / \cdot$ & قصل -بستى جامع -زرد \\
\hline & & & $|\Delta| / V Y$ & $91 / 99$ & قصد -بستخى جامع -قرمز \\
\hline \multirow[t]{2}{*}{$\cdot / 19 \mathrm{~V}$} & 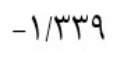 & 11 & $|r| / F D$ & $\mid \cdot \boldsymbol{c} / \cdot \Delta$ & قصل -بستخى جامع -سبز \\
\hline & & & Ir/VD & 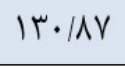 & قصل -بستخى جامع -آبى \\
\hline
\end{tabular}

${ }^{12}$ Intentional actions

${ }^{13}$ Voluntary 


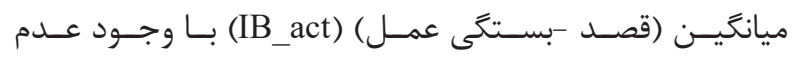

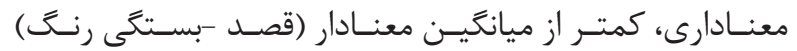

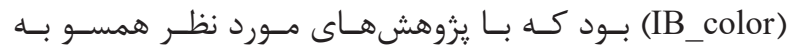

نظـــر مىرســد.

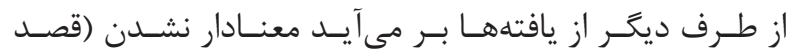

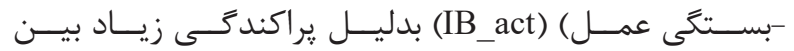

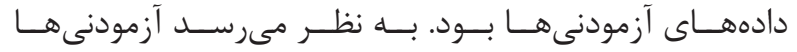

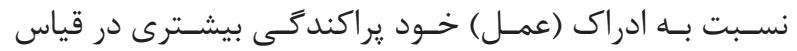

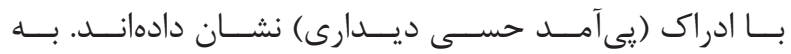

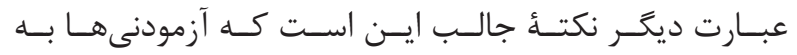

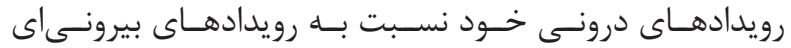

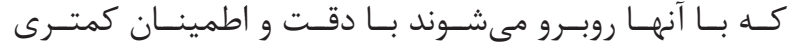

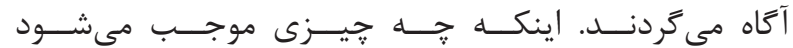

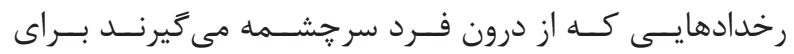

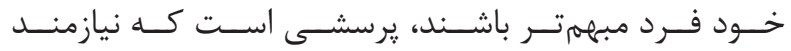

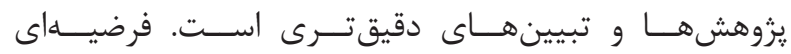

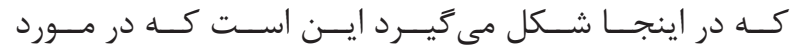

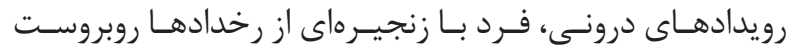

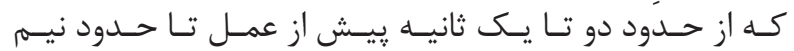

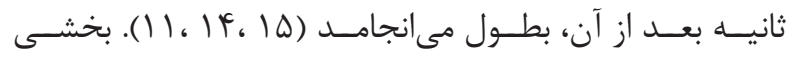

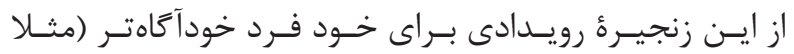

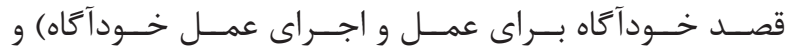

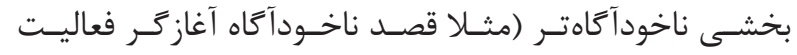

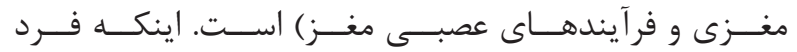

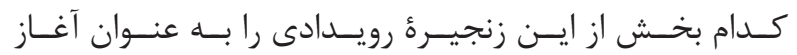

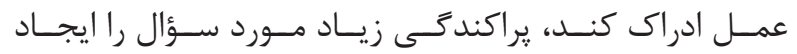

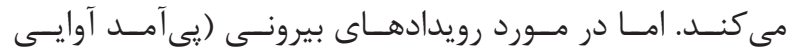

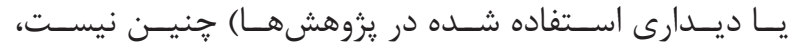

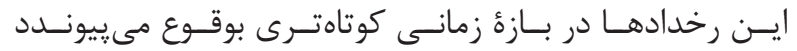

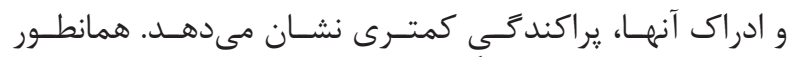

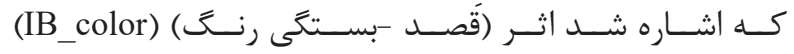

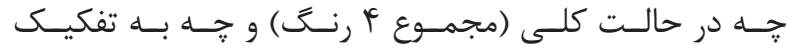

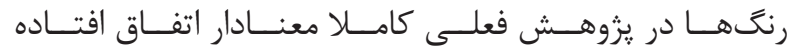

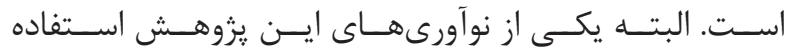

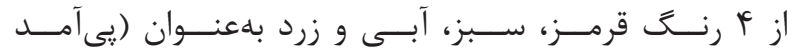

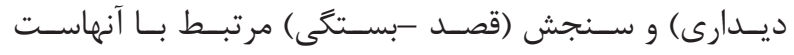

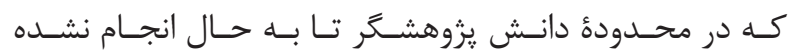

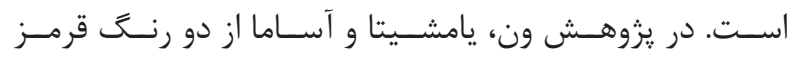

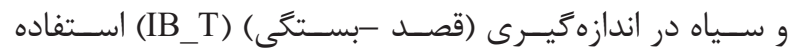

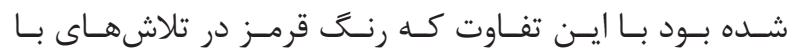

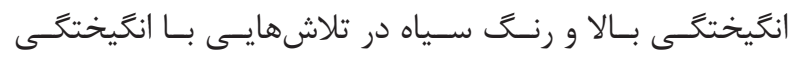

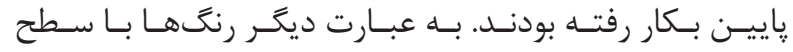

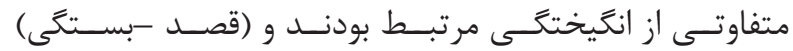

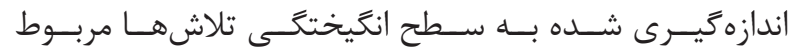

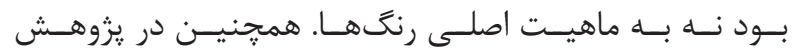

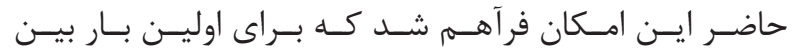

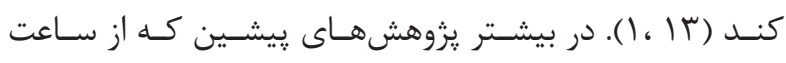

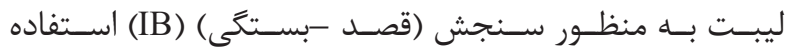

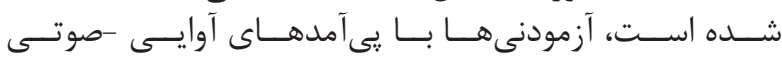

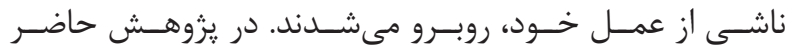

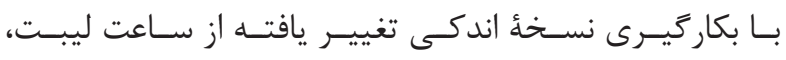

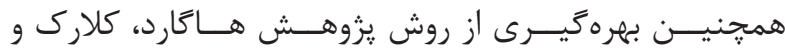

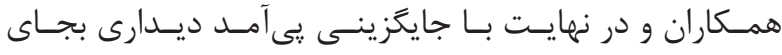

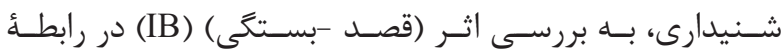

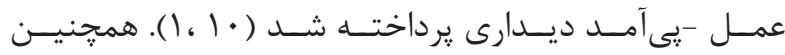

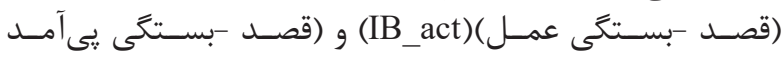

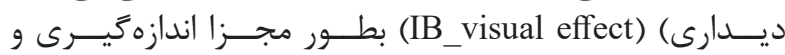

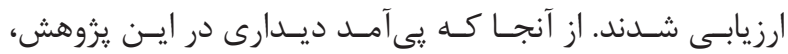

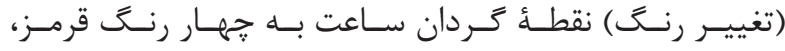

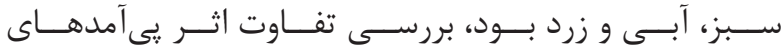

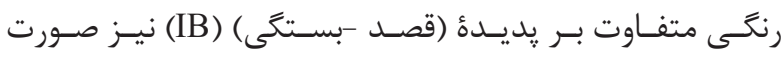

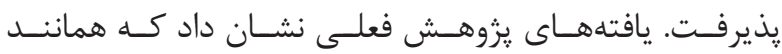

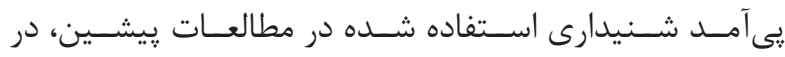

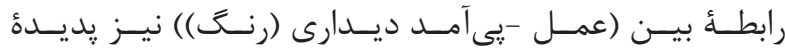

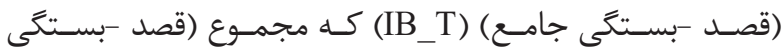

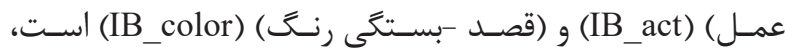

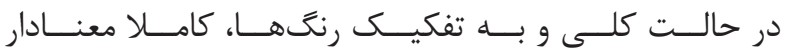

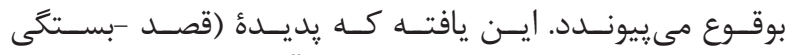

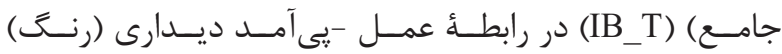

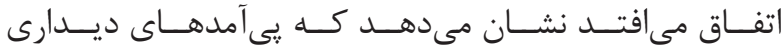

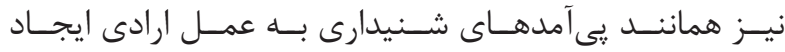

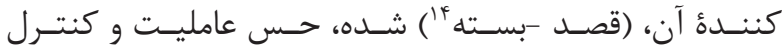

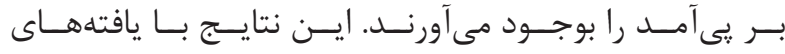

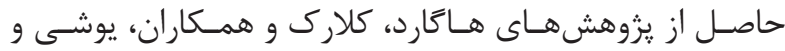

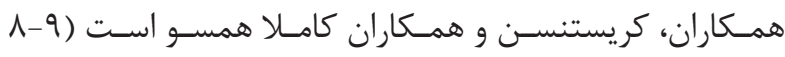

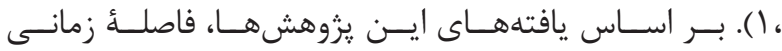

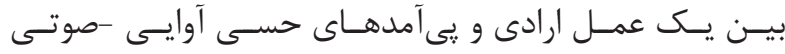

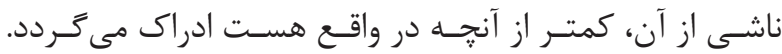

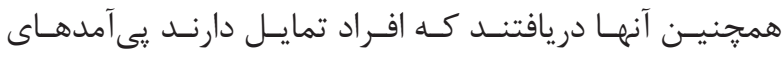

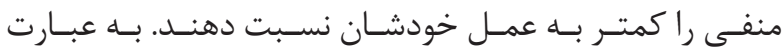

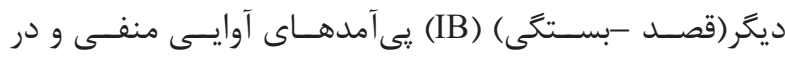

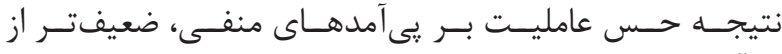

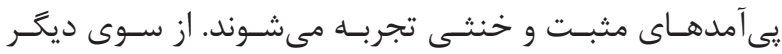

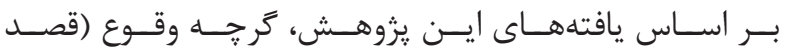

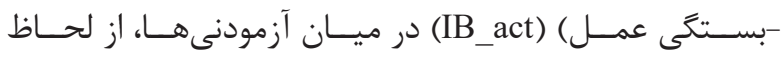

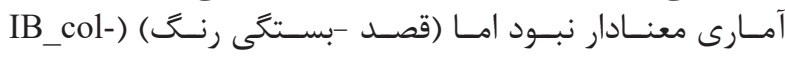

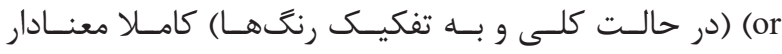

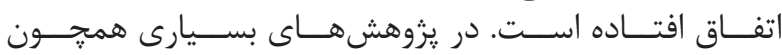

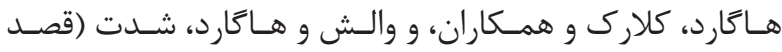

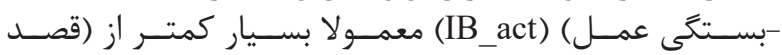

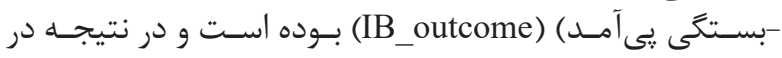

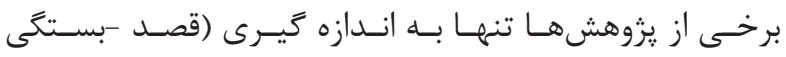

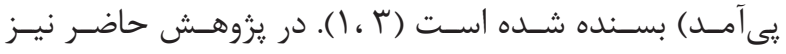

\footnotetext{
${ }^{14}$ intentionally bind
} 


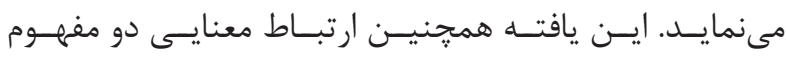

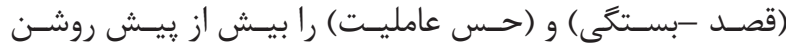

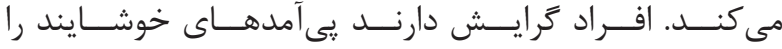

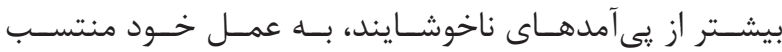

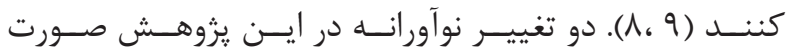

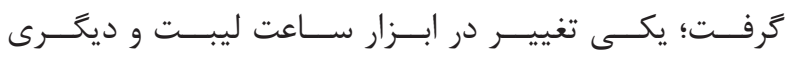

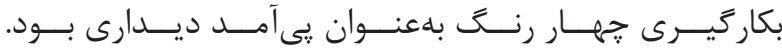

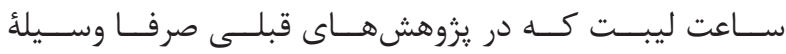

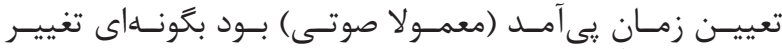

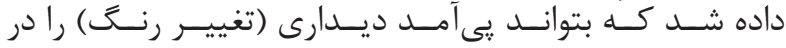

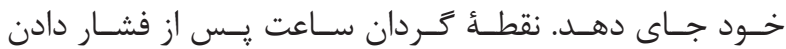

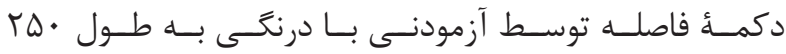

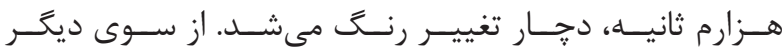

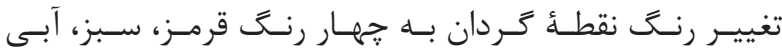

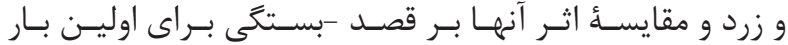

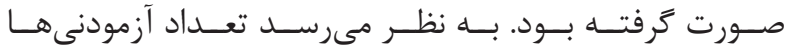

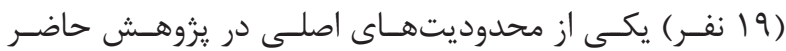

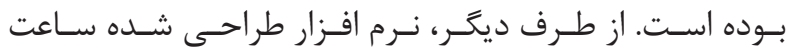

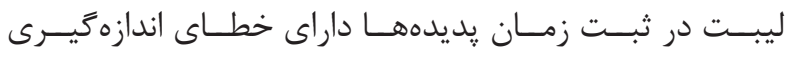

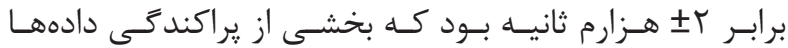

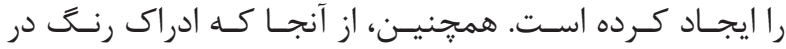

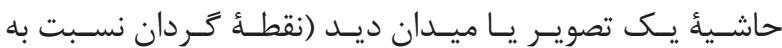

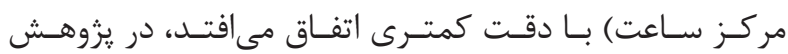

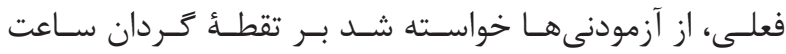

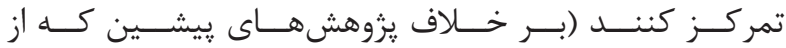

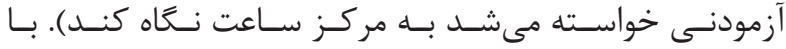

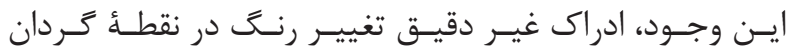

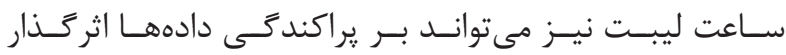

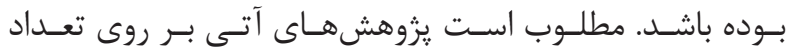

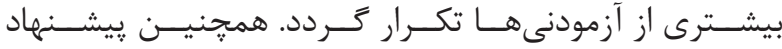

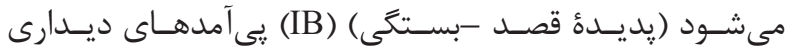

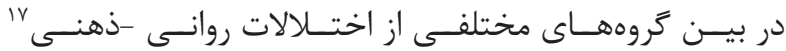

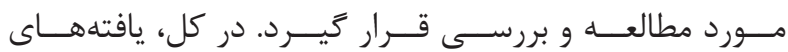

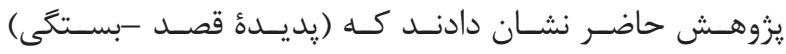

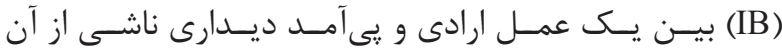

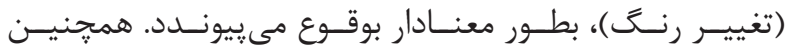

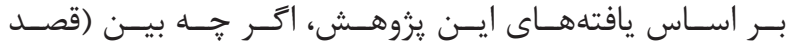

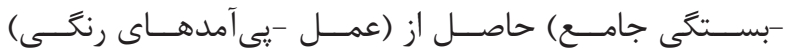

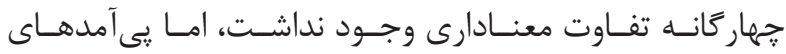

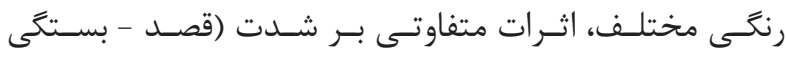

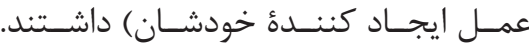

1. Haggard P, Clark S, Kalogeras J. Voluntary action and conscious awareness. Nature Neuroscience. 2002; 5(4): 3825.

2. Haggard P. Human volition: towards a neuroscience of will. Nature Reviews Neuroscience. 2008; 9(12): 934-46.

${ }^{15}$ Neuroscience

${ }^{16}$ retrospective

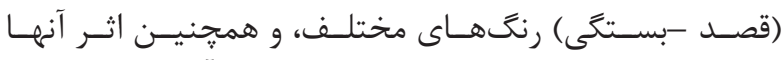

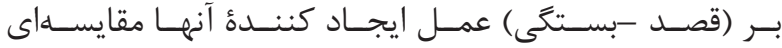

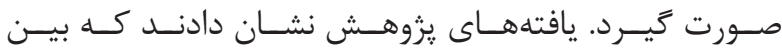

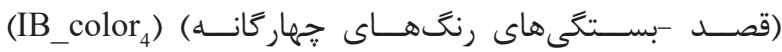

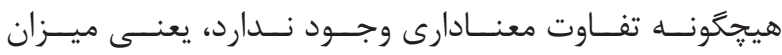

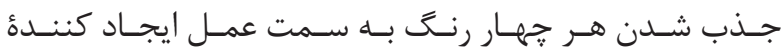

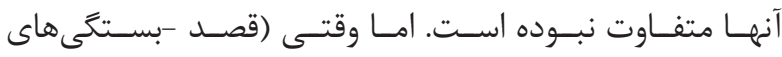

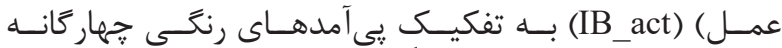

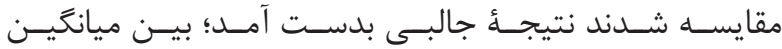

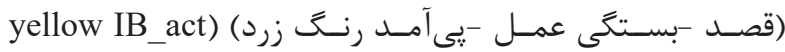

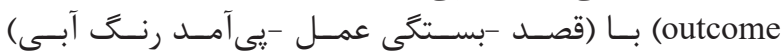

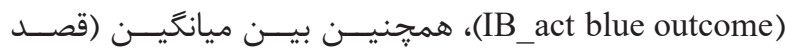

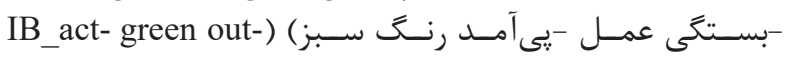

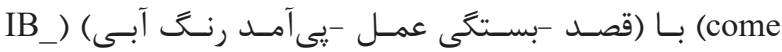

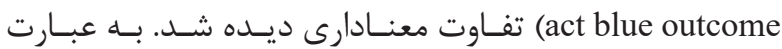

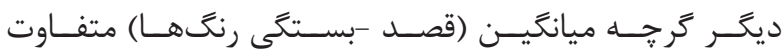

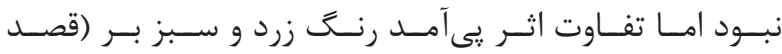

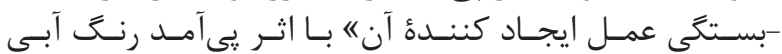

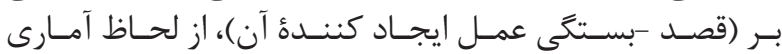

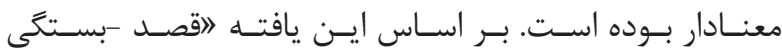

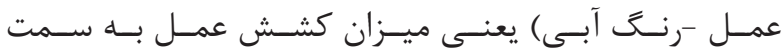

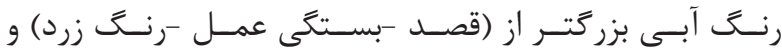

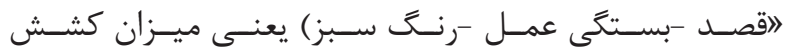

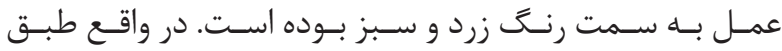

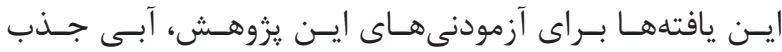

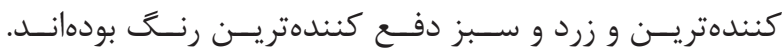

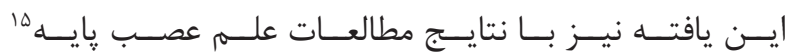

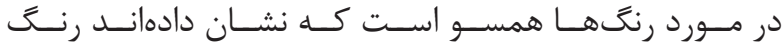

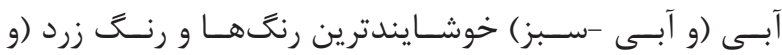

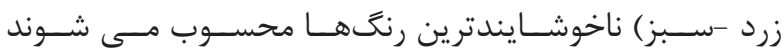

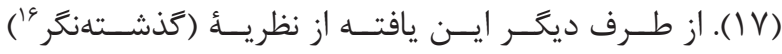

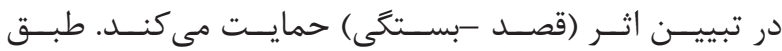

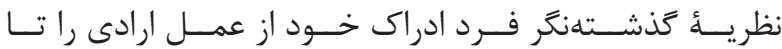

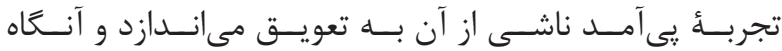

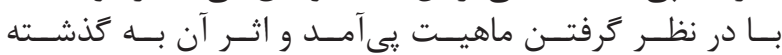

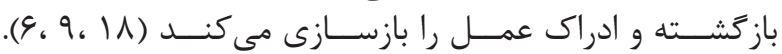

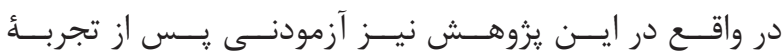

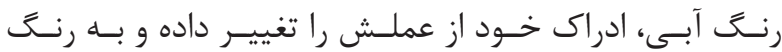

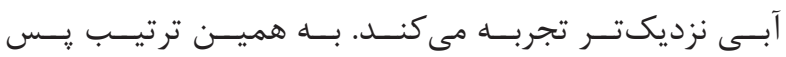

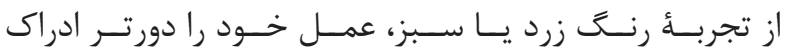

منابع

3. Walsh E, Haggard P. Action, prediction, and temporal awareness. Acta psychologica. 2013; 142(2): 220-9.

4. Haggard P, Aschersleben G, Gehrke J, Prinz W. Action, binding and awareness. Common mechanisms in

${ }^{17}$ Mental Disorders 


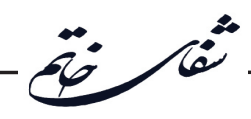

perception and action: Oxford University Press; 2002. p. 266-85.

5. Moore J, Haggard P. Awareness of action: Inference and prediction. Consciousness and cognition. 2008; 17(1): 13644 .

6. Moore JW, Obhi SS. Intentional binding and the sense of agency: a review. Consciousness and cognition. 2012; 21(1): 546-61.

7. Buehner MJ, Humphreys GR. Causal Binding of Actions to Their Effects. Psychological Science. 2009; 20(10): 1221-8.

8. Yoshie M, Haggard P. Negative emotional outcomes attenuate sense of agency over voluntary actions. Current Biology. 2013; 23(20): 2028-32.

9. Christensen J, Yoshie M, Di Costa S, Haggard P. Emotional valence, sense of agency and responsibility: A study using intentional binding. Consciousness and cognition. 2016; 43: 1-10.

10. Libert B, Gleason C, Wright E, Pearl D. Time of conscious intention to act in relation to onset of cerebral activity (readiness-potential). Brain. 1983; 106: 623-42.

11. Libet B, Wright Jr EW, Gleason CA. Preparationor intention-to-act, in relation to pre-event potentials recorded at the vertex. Electroencephalography and clinical Neurophysiology. 1983; 56(4): 367-72.
12. Libet B. Theory and evidence relating cerebral processes to conscious will. Behavioral and Brain Sciences. $1985 ; 8(4)$ : 558-66.

13. Obhi SS, Hall P. Sense of agency and intentional binding in joint action. Experimental brain research. $2011 ; 211(3-4): 655$.

14. Kornhuber HH, Deecke L. Hirnpotentialänderungen bei Willkürbewegungen und passiven Bewegungen des Menschen: Bereitschaftspotential und reafferente Potentiale. Pflüger's Archiv für die gesamte Physiologie des Menschen und der Tiere. 1965; 284(1): 1-17.

15. Deecke L, Grözinger B, Kornhuber H. Voluntary finger movement in man: Cerebral potentials and theory. Biological cybernetics. 1976; 23(2): 99-119.

16. Wen W, Yamashita A, Asama H. The influence of action-outcome delay and arousal on sense of agency and the intentional binding effect. Consciousness and cognition. 2015; 36: 87-95.

17. Valdez P, Mehrabian A. Effects of color on emotions. Journal of experimental psychology: General. 1994; 123(4): 394.

18. Moore JW, Haggard P. Intentional binding and higher order agency experience. Consciousness and cognition. 2010; 19(1): 490-1. 